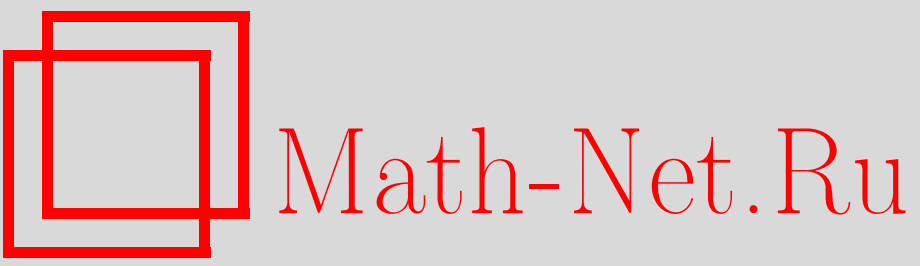

А. Н. Васильев, Парные корреляции в многокомпонентной анизотропной жидкости, ТМФ, 2003, том 135, номер 2, 315-321

DOI: https://doi.org/10.4213/tmf193

Использование Общероссийского математического портала Math-Net.Ru подразумевает, что вы прочитали и согласны с пользовательским соглашением

http://www.mathnet.ru/rus/agreement

Параметры загрузки:

IP: 54.174 .149 .18

26 апреля 2023 г., 13:24:36 


\title{
ПАРНЫЕ КОРРЕЛЯЦИИ В МНОГОКОМПОНЕНТНОЙ АНИЗОтРОПнОЙ ЖИДКОСТИ
}

\begin{abstract}
Рассмотрена многокомпонентная жидкая анизотропная система. При нахождении асимптотических выражений для парных корреляционных функций учтен первый пространственный момент прямой корреляционной функции. В этом приближении найдены парные корреляционные функции, которые описывают поведение системы не только в окрестности критического состояния, но и в некритической области. Показано, что наличие анизотропии приводит к изменению критических параметров по сравнению с изотропной системой.
\end{abstract}

Ключевые слова: многокомпонентная система, корреляционная функция, критическая гиперповерхность.

Статистическая теория жидкостей базируется в значительной степени на вычислении выражений для корреляционных функций. Это справедливо и при исследовании критического поведения жидких систем. Поэтому задача расчета парных корреляций часто имеет первостепенное значение.

Рассмотрим многокомпонентную жидкую систему. Для такой системы запишем уравнение Орнштейна-Цернике (ОЦ) [1] для парных $h_{i j}(\vec{r})$ и прямых $c_{i j}(\vec{r})$ корреляционных функций в виде

$$
h_{i j}(\vec{r})=c_{i j}(\vec{r})+\sum_{k=1}^{N}\left\langle\rho_{k}\right\rangle \int c_{i k}\left(\vec{r}_{1}\right) h_{k j}\left(\left|\vec{r}-\vec{r}_{1}\right|\right) d \vec{r}_{1}
$$

где $\left\langle\rho_{k}\right\rangle$ - средние плотности компонентов, число которых равно $N$, и соответственно индексы $i, j, k=1, \ldots, N$. В уравнении (1) удобно перейти к нормированным на плотности функциям [2]

$$
\begin{aligned}
h_{i j}(\vec{r}) & =\frac{G_{i j}(\vec{r})}{\sqrt{\left\langle\rho_{i}\right\rangle\left\langle\rho_{j}\right\rangle}}, \\
c_{i j}(\vec{r}) & =\frac{F_{i j}(\vec{r})}{\sqrt{\left\langle\rho_{i}\right\rangle\left\langle\rho_{j}\right\rangle}} .
\end{aligned}
$$

* Киевский национальный университет, Киев, Украина. E-mail: vasilal@phys.univ.kiev.ua 
Тогда уравнение (1) может быть сведено к матричному интегральному уравнению, формально совпадающему с уравнением ОЦ для однокомпонентной системы,

$$
\hat{g}(\vec{r})=\hat{f}(\vec{r})+\int \hat{f}\left(\vec{r}_{1}\right) \hat{g}\left(\left|\vec{r}-\vec{r}_{1}\right|\right) d \vec{r}_{1} .
$$

В последнем уравнении элементами матрицы $\hat{g}(\vec{r})$ ранга $N$ являются парные корреляционные функции $G_{i j}(\vec{r})$, а матрицы $\hat{f}(\vec{r})$ - прямые корреляционные функции $F_{i j}(\vec{r})$. Обе матрищы, очевидно, являются симметричными.

Для обычных растворов (под обычными здесь понимаются жидкости, взаимодействие в которых носит локальный характер) прямые корреляционные функции очень быстро убывают с расстоянием. Этим фактом можно воспользоваться для того, чтобы разложить парные корреляционные функции в ряд по вектору интегрирования [1]. В случае изотропных систем, когда корреляционные функции зависят только от расстояния между точками и не зависят от направления, в силу симметрии задачи ненулевыми будут только четные пространственные моменты прямых корреляционных функций [1], [3]. Если система находится во внешнем поле (или является анизотропной в силу иных причин), то, строго говоря, отличными от нуля будут и нечетные моменты. Рассмотрим приближение трех моментов, когда в разложении под интегралом оставляются слагаемые, пропорциональные нулевым

$$
C_{i j}^{(0)}=\int F_{i j}(\vec{r}) d \vec{r}
$$

первым

$$
\vec{C}_{i j}^{(1)}=\int F_{i j}(\vec{r}) \vec{r} d \vec{r}
$$

и вторым

$$
C_{i j}^{(2)}=\frac{1}{6} \int F_{i j}(\vec{r}) r^{2} d \vec{r}
$$

пространственным моментам прямых корреляционных функций. Вторые моменты взяты в изотропном приближении, т.е. при выгислении соответствующего интеграла предполагается эквивалентность трех различных направлений. В обшем случае это не так [4]. Однако можно ожидать, что на фоне отличного от нуля первого пространственного момента анизотропию можно не принимать во внимание.

Таким образом, имеем

$$
\hat{g}(\vec{r})=\hat{f}(\vec{r})+\widehat{C}^{(0)} \hat{g}(\vec{r})-\widehat{C}^{(1)} \nabla \hat{g}(\vec{r})+\widehat{C}^{(2)} \Delta \hat{g}(\vec{r}),
$$

причем элементами матрицы (ранга $N$ ) первых моментов являются векторы (пространственные, размерности 3), а соответствуюшее произведение элементов матриц в этом случае понимается в смысле скалярного произведения векторов. Производная от матрицы есть матрица, элементами которой являются производные от соответствующих элементов исходной матришы. 
Сделаем одно замечание. При переходе от интегрального уравнения (4) к дифференциальному (8) нарушается симметрия системы. Связано это с введением матриц пространственных моментов, элементы которых, строго говоря, для разных матриц являются зависимыми в том смысле, что вычисляются через одни и те же прямые корреляционные функции. Поэтому, чтобы не нарушать самосогласованности задачи, необходимо потребовать, чтобы матрицы пространственных моментов коммутировали между собой [2]. Последнее налагает определенные ограничения на элементы матриц пространственных моментов, и не все они, как было отмечено выше, будут независимы. В дальнейшем, если не оговорено противное, будем считать, что все рассматриваемые матрицы являются симметричными и коммутируют друг с другом.

Для упрошения последующего анализа выберем систему координат так, чтобы ось $z$ совпадала с направлением поля (т.е. направлением анизотропии). Тогда в элементах матришы первых пространственных моментов отличными от нуля будут только $z$-компоненты векторов. В этом случае

$$
\widehat{C}^{(1)} \nabla \hat{g}(\vec{r}) \equiv \widehat{C}_{z}^{(1)} \frac{\partial \hat{g}(\vec{r})}{\partial z} .
$$

Нижний индекс в матрице $\widehat{C}_{z}^{(1)}$ указьвает на то, что эта матрица содержит теперь только $z$-компоненты векторов первых моментов, т.е. ее элементами являются скалярные величины.

Для решения уравнения (8) удобно представить корреляционные функции в виде

$$
\begin{aligned}
& \hat{g}(\vec{r})=\widehat{\Psi}(\vec{r}) \widehat{U}(\hat{\gamma} z), \\
& \hat{f}(\vec{r})=\widehat{\Phi}(\vec{r}) \widehat{U}(\hat{\gamma} z),
\end{aligned}
$$

где матричная функция $\widehat{U}(\hat{\gamma} z)$ удовлетворяет уравнению

$$
\frac{\partial \widehat{U}(\hat{\gamma} z)}{\partial z}=\hat{\gamma} \widehat{U}(\hat{\gamma} z)
$$

a $\hat{\gamma}$ есть матрица-параметр, который выбирается из условия обрашения градиентного слагаемого в нуль. В данном случае, как несложно убедиться, следует положить

$$
\hat{\gamma}=\frac{1}{2}\left[\widehat{C}^{(2)}\right]^{-1} \widehat{C}_{z}^{(1)}
$$

Матрица $\widehat{U}(\hat{\gamma} z)$, удовлетворяюшая уравнению (12), в обшем случае выбирается неоднозначно. Будем искать ее симметричной и требовать, чтобы при $z=0$ (или $\hat{\gamma} \rightarrow \widehat{O}$, где $\widehat{O}$ - нуль-матрица) матрица $\widehat{U}$ равнялась бы единичной матрище. Формально $\widehat{U}(\hat{\gamma} z)$ можно представить в виде операторной экспоненты, причем последняя, как обычно, понимается в смысле ряда:

$$
\widehat{U}(\hat{\gamma} z)=\sum_{n=0}^{\infty} \frac{\hat{\gamma}^{n} z^{n}}{n !} .
$$


Такое представление может быть использовано при численных расчетах, однако для аналитического анализа удобнее записать $\widehat{U}(\hat{\gamma} z)$ в виде разложения по умноженным на $z$ экспонированным собственным числам матрицы $\hat{\gamma}$,

$$
\widehat{U}(\hat{\gamma} z)=\sum_{k=1}^{N} \hat{\alpha}_{k} e^{\lambda_{k} z}
$$

где $\lambda_{k}$ - собственные числа матрицы $\hat{\gamma}$, а столбцы симметричных вырожденных матриц $\hat{\alpha}_{k}$ пропорциональны собственному вектору $\vec{\xi}_{k}$ матрицы $\hat{\gamma}$, соответствующему собственному числу $\lambda_{k}$,

$$
\hat{\gamma} \vec{\xi}_{k}=\lambda_{k} \vec{\xi}_{k}
$$

и соответственно

$$
\hat{\alpha}_{k}=a_{1}^{(k)} \vec{\xi}_{k} \oplus a_{2}^{(k)} \vec{\xi}_{k} \oplus \cdots \oplus a_{N}^{(k)} \vec{\xi}_{k}
$$

Условие симметричности позволяет определить матрищы $\hat{\alpha}_{k}$ с точностью до одного обшего множителя. Поскольку при $z=0$ имеем $\widehat{U}=\hat{I}$, т.е.

$$
\sum_{k=1}^{N} \hat{\alpha}_{k}=\hat{I}
$$

где $\hat{I}$ - единичная матрица ранга $N$, то матрицы $\hat{\alpha}_{k}$ определяются однозначно.

Таким образом, подставляя выражения (10), (11) в уравнение (8) и принимая во внимание уравнение (12), получим

$$
\widehat{\Psi}(r)=\widehat{\Phi}(r)+\left[\widehat{C}^{(0)}-\frac{1}{4}\left[\widehat{C}^{(2)}\right]^{-1}\left(\widehat{C}_{z}^{(1)}\right)^{2}\right] \widehat{\Psi}(r)+\widehat{C}^{(2)} \Delta \widehat{\Psi}(r)
$$

В более традиционной форме, напоминающей дифференциальное уравнение ОЦ (но только теперь оно еше и матричное), уравнение (19) имеет вид

$$
\Delta \widehat{\Psi}(r)-\left[\widehat{C}^{(2)}\right]^{-1}\left[\hat{I}-\widehat{C}^{(0)}+\frac{1}{4}\left[\widehat{C}^{(2)}\right]^{-1}\left(\widehat{C}_{z}^{(1)}\right)^{2}\right] \widehat{\Psi}(r)=-\left[\widehat{C}^{(2)}\right]^{-1} \widehat{\Phi}(r) .
$$

После преобразования Фурье для гармоник корреляционных функций получаем соотношение

$$
\widehat{\Psi}(k)=\left[\hat{I} k^{2}+\left[\widehat{C}^{(2)}\right]^{-1}\left(\hat{I}-\widehat{C}^{(0)}+\frac{1}{4}\left[\widehat{C}^{(2)}\right]^{-1}\left(\widehat{C}_{z}^{(1)}\right)^{2}\right)\right]^{-1}\left[\widehat{C}^{(2)}\right]^{-1} \widehat{\Phi}(k) .
$$

Для поиска асимптотического выражения для парной корреляционной функции в уравнении (21) следует либо задать прямую корреляционную функцию, либо искать решение исходя из некоторых дополнительных соображений, например предположить, что парная корреляционная функция должна быть короткодействующей даже в критическом состоянии (для систем с локальными прямыми взаимодействиями, которые здесь рассматриваются). Модельные функции, как правило, используют непосредственно в 
исходном интегральном уравнении. Нахождение парных функций из дифференциального уравнения на феноменологической основе достаточно громоздко [1]. Однако оказывается, что те же результаты могут быть получены, если в дифференциальном уравнении в качестве нулевого приближения для прямых корреляционных функций взять $\delta$-функцию Дирака [4]. Положим $\hat{f}(\vec{r})=\widehat{C}^{(0)} \widehat{U}(\hat{\gamma} z) \delta(\vec{r})$. Тогда из уравнения $(21)$ получим

$$
\widehat{\Psi}(k)=\left[\hat{I} k^{2}+\left[\widehat{C}^{(2)}\right]^{-1}\left(\hat{I}-\widehat{C}^{(0)}+\frac{1}{4}\left[\widehat{C}^{(2)}\right]^{-1}\left(\widehat{C}_{z}^{(1)}\right)^{2}\right)\right]^{-1}\left[\widehat{C}^{(2)}\right]^{-1} \widehat{C}^{(0)} .
$$

Введем обозначение

$$
\widehat{\Theta}=\left[\widehat{C}^{(2)}\right]^{-1}\left(\hat{I}-\widehat{C}^{(0)}+\frac{1}{4}\left[\widehat{C}^{(2)}\right]^{-1}\left(\widehat{C}_{z}^{(1)}\right)^{2}\right)
$$

Если $\kappa_{i}^{2}, i=1, \ldots, N,-$ собственные числа матрищы $\widehat{\Theta}$ (предполагаем, что все они положительные и различные), то матрицу $\left[\hat{I} k^{2}+\widehat{\Theta}\right]^{-1}$ удобно представить в виде

$$
\left[\hat{I} k^{2}+\widehat{\Theta}\right]^{-1}=\sum_{i=1}^{N} \frac{\widehat{S}_{i}}{k^{2}+\kappa_{i}^{2}}
$$

где матрицы спектрального разложения

$$
\widehat{S}_{i}=\prod_{\substack{j=1 \\ j \neq i}}^{N} \frac{\hat{I} \kappa_{j}^{2}-\widehat{\Theta}}{\kappa_{j}^{2}-\kappa_{i}^{2}}
$$

Таким образом,

$$
\widehat{\Psi}(k)=\sum_{i=1}^{N} \frac{\widehat{S}_{i}\left[\widehat{C}^{(2)}\right]^{-1} \widehat{C}^{(0)}}{k^{2}+\kappa_{i}^{2}}
$$

Отсюда несложно получить, что

$$
\widehat{\Psi}(k)=\sum_{i=1}^{N} \frac{\widehat{S}_{i}\left[\widehat{C}^{(2)}\right]^{-1} \widehat{C}^{(0)} e^{-\kappa_{i} r}}{4 \pi r} .
$$

Матрица парных корреляционных функций анизотропной системы будет согласно (10) иметь вид

$$
\widehat{\Psi}(k)=\sum_{i=1}^{N} \frac{\widehat{S}_{i}\left[\widehat{C}^{(2)}\right]^{-1} \widehat{C}^{(0)} \widehat{U}(\hat{\gamma} z) e^{-\kappa_{i} r}}{4 \pi r} .
$$

Таким образом, приходим к выводу, что парные корреляционные функции многокомпонентной системы представляются в виде линейной комбинации набора базисных функций

$$
\phi_{i j}(\vec{r})=\frac{e^{\lambda_{j} z-\kappa_{i} r}}{r} .
$$


Полученные результаты свидетельствуют о том, что в плоскости, перпендикулярной направлению анизотропии (т.е. при $z=0$ ), парные коррелящионные функции определяются формулой (27) и формально совпадают с парными корреляционными функциями изотропной системы. Однако в данном случае, в отличие от анизотропной системы, собственные числа $\kappa_{i}^{2}$ зависят от первых пространственных моментов. Поскольку известно, что критическое состояние характеризуется аномальным возрастанием взаимодействия флуктуаций, то следует ожидать, что на уровне корреляционных функций это проявится как обрашение в нуль одного из показателей в экспонентах (т.е. собственных чисел). В случае изотропной многокомпонентной системы такими показателями являются собственные числа матрицы $\left[\widehat{C}^{(2)}\right]^{-1}\left(\hat{I}-\widehat{C}^{(0)}\right)$. Отсюда следует вывод, что в анизотропных системах имеет место изменение критических параметров по сравнению с изотропными. Проведем оценку таких изменений.

В этом случае удобно применить геометрический подход, развитый в работе [5]. Положение системы характеризуется точкой в пространстве термодинамических переменных. Для системы из $N$ компонентов размерность такого пространства равна $N+1$. Критическая область в пространстве термодинамических переменных является гиперповерхностью размерности $N-1$. Направление подхода к этой гиперповерхности (другими словами, способ фиксации термодинамических переменных) определяет характер термодинамических аномалий. Рассмотрим, например, как анизотропия влияет на сдвиг критической температуры (при фиксированных прочих параметрах). В теории скейлинга критические аномалии принято характеризовать критическими индексами. Не касаясь в данном случае вопроса о связи таких индексов со способом выбора независимых термодинамических переменных, запишем зависимость для одного из собственных чисел матрицы $\left[\widehat{C}^{(2)}\right]^{-1}\left(\hat{I}-\widehat{C}^{(0)}\right)$ в виде

$$
\kappa_{i}^{*}=\varepsilon_{i}^{*}\left(\frac{T-T_{\mathrm{c}}^{*}}{T_{\mathrm{c}}^{*}}\right)^{\Delta},
$$

где $\varepsilon_{i}^{*}$ - размерная амплитуда, не зависящая от температуры $T, T_{\mathrm{c}}^{*}$ - критическая температура изотропной системы (зависяшая, как отмечалось, от $N-2$ независимых фиксированных параметров), $\Delta$ - критический индекс, определяемый способом выбора упомянутых независимых переменных. Предположим далее, что при "включении" поля характер зависимости (30) не меняется, т.е. для одного из собственных чисел матрицы $\widehat{\Theta}$ справедливо представление

$$
\kappa_{i}=\varepsilon_{i}\left(\frac{T-T_{\mathrm{c}}}{T_{\mathrm{c}}}\right)^{\Delta} .
$$

Однако теперь критическая температура анизотропной системы $T_{\mathrm{c}}$ отличается от $T_{\mathrm{c}}^{*}$. Представим обрашаюшееся в нуль собственное число матрицы $\widehat{\Theta}$ в виде

$$
\kappa_{i}^{2}=\left(\kappa_{i}^{*}\right)^{2}+\delta \kappa_{i}^{2}(\hat{\gamma}),
$$

где добавка $\delta \kappa_{i}^{2}(\hat{\gamma})$ обусловлена анизотропией системы. Зависимость этой добавки от матрицы $\hat{\gamma}$ следует понимать в том смысле, что она является функцией инвариантов упомянутой матрищы, а значит, ее собственных чисел. Следует заметить, что в обшем случае $\delta \kappa_{i}^{2}(\hat{\gamma})$ зависит также и от $\widehat{\Theta}$. 
Не затрагивая вопроса о явном виде $\delta \kappa_{i}^{2}(\hat{\gamma})$, можно записать, что в критической точке изотропной системы

$$
T_{\mathrm{c}}=\frac{T_{\mathrm{c}}^{*}}{1+\left(\delta \kappa_{i}(\hat{\gamma}) \varepsilon_{i}^{-1}\right)^{1 / \Delta}} .
$$

Уравнение (33) записано для критической температуры при фиксированных прочих параметрах, а критический индекс $\Delta$ во многом определяется именно выбором и способом фиксации последних. Поэтому уравнение (33) определяет закон трансформации критической гиперповерхности при возникновении анизотропии.

Полученные результаты при $N=2$ хорошо согласуются с данными для двухкомпонентных систем. Это, в первую очередь, относится к структуре корреляционных функций и сдвигам критических параметров [4]. Обобщение таких результатов может стать серьезной предпосылкой для исследования более сложных систем, например жидкостей, содержаших примеси различного типа.

\section{Список литературы}

[1] А. Мюнстер. Теория фолуктуаций. В сб.: Термодинамика необратимых процессов. Ред. Д. Н. Зубарев. М.: ИЛ, 1962. С. 36.

[2] А. Н. Васильев, А. В. Чальй. Журн. физ. достиж. 2000. Т. 4. С. 266.

[3] В. М. Съсоев, А. В. Чальй, Л. М. Черненко. ТМФ. 1975. Т. 22. С. 135.

[4] A. V. Chalyi et al. Cond. Mat. Phys. 2000. V. 3. P. 335.

[5] R. B. Griffiths, J. C. Wheeler. Phys. Rev. A. 1970. V. 2. P. 1047.

Поступила в редакцию 22.V.2002 г. 